\title{
Microsymposia
}

MS.51.1

Acta Cryst. (2008). A64, C91

\section{Joint use of SAXS and SANS with high resolution methods for macromolecular solutions}

\section{Dmitri I. Svergun $^{1,2}$}

${ }^{1}$ EMBL, ${ }^{2}$ Institute of Crystallography, Russian Academy of Sciences, Leninsky pr. 59, 117333 Moscow, E-mail:svergun@embl-hamburg.de

Small-angle scattering of X-rays and neutrons (SAS) allows one to study the structure of native particles in nearly physiological solutions and to analyse structural changes in response to variations in external conditions. The scattering data bear information about the overall shape and internal structure at a resolution of 1-2 nm. The method is applicable to a broad range of sizes, from individual macromolecules to multi-domain proteins and large macromolecular assemblies. Recent progress in instrumentation and especially development of novel data analysis methods [1] significantly enhanced resolution and reliability of structural models provided by the technique and made SAS a useful complementary tool to high resolution methods, including large scale structural studies. In particular, rapid validation of crystallographic models in solution, identification of biologically active oligomers and addition of missing fragments to high resolution models are possible. For macromolecular complexes, quaternary structure can be analyzed in terms of rigid body movements/rotations of high resolution models of the individual subunits of domains. Advanced applications of SAS to macromolecular solutions will be presented including, in particular, ab initio low resolution structure determination, rigid body refinement, contrast variation studies of complexes and quantitative analyses of flexible macromolecules.

[1] Petoukhov, M.V. \& Svergun, D. I. (2007) Curr Opin Struct Biol. $17,562-571$

Keywords: small-angle scattering, macromoleclar structure, rigid body refinement

\section{MS.51.2}

Acta Cryst. (2008). A64, C91

\section{Additivity, redundancy, and complementarity between structural information from NMR and SAXS data}

Masaki Kojima ${ }^{1}$, Yasumasa Morimoto ${ }^{2}$, Takashi Nakagawa ${ }^{2}$, Shigeru Yanagi $^{2}$, Hiroshi Kihara ${ }^{3}$, Takamasa Nonaka ${ }^{1}$

${ }^{1}$ Iwate Medical University, School of Pharmacy, 2-1-1 Nishitokuta, Yahaba, Iwate, 028-3694, Japan, ${ }^{2}$ Tokyo University of Pharmacy and Life Science, 1432-1 Horinouchi, Hachioji, Tokyo, 192-0392, Japan, ${ }^{3}$ Kansai Medical University, 18-89 Uyama-Higashi, Hirakata, Osaka, 573-1136, Japan, E-mail:mkojima@iwate-med.ac.jp

At present protein structure in solution is determined by restrained molecular dynamics with distance restraints mainly derived from NMR. Although the small-angle X-ray scattering (SAXS) method also confers the structural information, its content is too small to determine the structure by itself. We previously developed a new algorithm that refines the protein structure by restrained molecular dynamics with SAXS constraints ${ }^{1}$. In the present study we performed the protein structure calculation by restrained molecular dynamics with both NMR and SAXS constraints, in order to elucidate the essential structural information that defines the protein architecture. We used RNase T1 as a model protein, which has already been determined by NMR alone ${ }^{2}$. At first we added SAXS constraints $(\mathrm{h}<$ $0.3 \AA^{-1}$ ) into the original NMR-derived restraints for the calculation. The quality of the structure ensemble was significantly increased. Next we removed the original NMR restraints randomly in order to estimate the redundancy among the NMR-derived information. The essential topology of the resultant structures was hardly changed until the restraints were reduced below the half. Then we added the SAXS constraints into the remaining NMR restraints to expect they could complement the lost structural information. However, the structure was not recovered properly. By removing various types of structural information exclusively from the original NMR data set, we investigated whether the SAXS constraints could complement some kinds of structural information. The results showed that the SAXS could complement the tertiary structure to some extent while it could not secondary structure.

1) Kojima et al. (2004) J. Appl. Cryst. 37, 103-109

2) Hatano et al. (2003) Biol. Chem. 384, 1173-1183

Keywords: SAXS, NMR, molecular dynamics

\section{MS.51.4}

Acta Cryst. (2008). A64, C91-92

\section{Time-resolved X-ray scattering studies on bacteriophage assemblies}

Hiro Tsuruta ${ }^{1}$, Roman Tuma ${ }^{2,3}$, Kenneth H French ${ }^{2}$, Peter E Prevelige ${ }^{2}$, Kelly K Lee ${ }^{4}$, Lu Gan ${ }^{4}$, Crystal Moyer ${ }^{5}$, James F Conway ${ }^{6}$, Robert L Duda ${ }^{5}$, Roger W Hendrix ${ }^{5}$, Alasdair C Steven ${ }^{7}$, John E Johnson ${ }^{4}$

${ }^{1}$ Stanford University, SSRL/SLAC, 2575 Sand Hill Rd MS69, Menlo Park, CA, 94025-7015, USA, ${ }^{2}$ University of Alabama at Birmingham, USA, ${ }^{3}$ Universtiy of Leeds, UK, ${ }^{4}$ The Scripps Research Institute, USA, ${ }^{5}$ University of Pittsburgh, USA, ${ }^{6}$ University of Pittsburgh School of Medicine, USA, ${ }^{7}$ National Institute of Health, USA, E-mail : tsuruta@slac. 


\section{Microsymposia}

stanford.edu

The solution x-ray scattering technique permits studies of biological macromolecules in near physiological conditions, which are not always compatible with high-resolution structural studies. Timeresolved solution scattering studies have been used to examine a number of different conformational changes as a function of time primarily at the tertiary and/or quaternary structure level. There exist a number of macromolecular systems which simply can not be contained within the crystallographic lattice structure. All virus and bacteriophage systems undergo complex assembly and maturation processes. Solution x-ray scattering and their time-resolved studies cover small proteins of several thousands of $\mathrm{Da}$ to multimega $\mathrm{Da}$ virus/phage particles, allowing structural studies of initial assembly processes as well as the late maturation processes. This talk will highlight the time-resolved study on the scaffolding protein-mediated assembly of P22 bacteriophage capsid and the maturation structural kinetics of HK97 bacteriophage capsid to illustrate how timeresolved solution scattering studies can complement crystallography and cryoelectron microscopy in structural virology. It will be shown that the scaffolding protein monomer-dimer assembly equilibrium controls the entire P22 capsid assembly process. In the case of HK97 maturation, the highly cooperative all-or-nothing structural transitions of 420 capsid proteins preveil in all steps so far been examined.

Keywords: small angle X-ray scattering, viral structure and function, time-resolved $\mathrm{x}$-ray analysis

\section{MS.51.5}

Acta Cryst. (2008). A64, C92

Real-time SAXS observation of assembly and disassembly dynamics of cyanobacterial clock proteins

\section{Shuji Akiyama $^{1,2}$, Atsushi Nohara ${ }^{3}$, Kazuki Ito ${ }^{2}$, Yuichiro Maeda ${ }^{4}$}

${ }^{1}$ Japan Science and Technology Agency, PRESTO, RIKEN SPring-8 Center, Harima Institute, Structural Biophysics Laboratory, 1-1-1, Kouto, Sayo, Hyogo, 679-5148, Japan, ${ }^{2}$ RIKEN SPring-8 Center, Harima Institute, 1-1-1, Kouto, Sayo, Hyogo 679-5148, Japan, ${ }^{3}$ Division of Biological Science, Graduate School of Science, Nagoya University, Furocho, Chikusa-ku, Nagoya 464-8602, Japan, ${ }^{4}$ Structural Biology Research Center, Graduate School of Science, Nagoya University, Furo-cho, Chikusa-ku, Nagoya 464-8601, Japan, E-mail:akishu@spring8.or.jp

Circadian clocks are the endogenous timing systems enabling a variety of living organisms to adapt daily alternation of environments. Cyanobacterium Synechococcus elongatus PCC 7942 is known to have an oscillator composed of three clock proteins termed KaiA, $\mathrm{KaiB}$, and KaiC. KaiA promotes the autophosphorylation of KaiC, whereas KaiB promotes the autodephosphorylation of KaiC. Three Kai proteins incubated in the presence of ATP are assembled and disassembled into heteromultimeric Kai complexes to effect a rhythmic change of the phosphorylation state of KaiC. To date, the crystal structure of the individual Kai protein has already been determined independently. However, a relationship between the assembly/disassembly dynamics and the KaiC phosphorylation cycle is still poorly understood because of the difficulty in unraveling the underlying mechanisms solely from the static molecular picture of individual clock components. We thus followed the assembly/ disassembly dynamics of a ternary mixture containing KaiA, $\mathrm{KaiB}$, and KaiC in real-time by using small-angle $\mathrm{x}$-ray scattering (SAXS) at beamline BL45XU of SPring-8. The scattering from the ternary mixture robustly oscillated with a period of approximately $24 \mathrm{~h}$, indicating a repeated assembly and disassembly of the Kai complexes. Based on the size and shape of the clock complexes, we will discuss the assembly/disassembly mechanism of the Kai oscillator.

Keywords: small-angle scattering, macromolecular complexes, dynamics thermodynamics of biomacromolecules

\section{MS.52.1}

Acta Cryst. (2008). A64, C92

\section{Novel cyclic salicylide derivatives: Guest inclusion and organo-gellation}

Koichi Tanaka $^{1}$, Satoshi Hayashi ${ }^{1}$, Mino R Caira ${ }^{2}$

${ }^{1}$ Kansai University, 3-3-35 Yamate-cho, Suita, Osaka, 564-8680, Japan, ${ }^{2}$ University of Cape Town, Rondebosch 7701, South Africa, E-mail : ktanaka@ipcku.kansai-u.ac.jp

Some novel tetra- and hexasalicylide derivatives (1 and 2) were synthesized. The tetrasalicylide derivatives (1b-1e) having a 5 -substituted halogen atom on the aromatic ring form organo-gels with several kinds of organic solvents, whereas the parent compound (1a) does not. In contrast, hexasalicylide derivatives $(2 a-2 b)$ form stable inclusion crystals with several organic guest molecules.
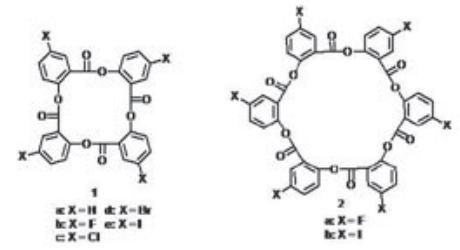

Keywords: inclusion chemistry, host-guest complexes, crystal structures

\section{MS.52.2}

Acta Cryst. (2008). A64, C92-93

\section{Porous material behaviour in non-porous crystals: A route to chemical reactions}

Lee Brammer ${ }^{1}$, Guillermo Minguez Espallargas ${ }^{1}$, Stefano Libri ${ }^{1}$, Jacco van de Streek ${ }^{2}$, Alastair J Florence ${ }^{3}$, Michela Brunelli ${ }^{4}$, Kenneth Shankland ${ }^{5}$, William I.F. David ${ }^{5}$

${ }^{1}$ Department of Chemistry, University of Sheffield, Brook Hill, Sheffield, South Yorkshire, S3 7HF, UK, ${ }^{2}$ Institute for Inorganic and Analytical Chemistry, Frankfurt University, 60438 Frankfurt am Main, Germany, ${ }^{3}$ Strathclyde Institute of Pharmacy and Biomedical Sciences, University of Strathclyde, 27 Taylor Street, Glasgow G4 0NR, Scotland, ${ }^{4}$ European Synchrotron Radiation Facility, 38042 Grenoble, France, ${ }^{5}$ ISIS Facility, Rutherford Appleton Laboratory, Chilton, Didcot, Oxon OX11 0QX, UK, E-mail:lee.brammer@sheffield.ac.uk

In porous crystalline materials an important emphasis is on interactions between the host framework and the guest molecules and upon transport of the guests in and out of the framework pores. Surprisingly, in a small number of examples reported to date, such transport can also occur in non-porous crystals leading to entrapment of small molecules.[1] In this presentation two examples will be discussed in which such transport behaviour in non-porous crystal leads to subsequent chemical reactions within the crystal. These reactions involve changes in hydrogen bonding and changes in coordination bonds. In the first case we explore the reversible uptake/ release of gaseous $\mathrm{HCl}$ leading to interconversion between squareplanar coordination complexes trans- $\left[\mathrm{CuCl}_{2}(3-\mathrm{Xpy})_{2}\right]$ and salts $(3-\mathrm{XpyH})_{2}\left[\mathrm{CuCl}_{4}\right]$ (3-Xpy $=3$-halopyridine) which has tetrahedral metal coordination geometry.[2,3] In the second, reversible uptake of 